\title{
Galectin-3 in Patients with Atrial Fibrillation Undergoing Radiofrequency Catheter Ablation
}

\author{
Jelena Kornej $^{1 \odot *}$, Josephin Schmidl ${ }^{1 \odot}$, Laura Ueberham ${ }^{1}$, Silke John ${ }^{1}$, \\ Sait Daneschnejad ${ }^{1}$, Borislav Dinov ${ }^{1}$, Gerhard Hindricks ${ }^{1}$, Volker Adams ${ }^{2}$, \\ Daniela Husser ${ }^{1}$, Andreas Bollmann ${ }^{1}$ \\ 1 Department of Electrophysiology, Heart Center, Leipzig, Germany, 2 Department of Cardiology, Heart \\ Center, Leipzig, Germany \\ จ These authors contributed equally to this work. \\ * jelena.kornej@gmx.de
}

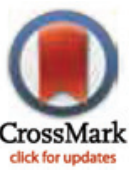

\section{Abstract}

\section{Background}

\section{Gopenaccess}

Citation: Kornej J, Schmidl J, Ueberham L, John S, Daneschnejad S, Dinov B, et al. (2015) Galectin-3 in Patients with Atrial Fibrillation Undergoing Radiofrequency Catheter Ablation. PLoS ONE 10(4): e0123574. doi:10.1371/journal.pone.0123574

Academic Editor: Randall Lee Rasmusson, University at Buffalo, UNITED STATES

Received: October 15, 2014

Accepted: March 4, 2015

Published: April 15, 2015

Copyright: @ 2015 Kornej et al. This is an open access article distributed under the terms of the Creative Commons Attribution License, which permits unrestricted use, distribution, and reproduction in any medium, provided the original author and source are credited.

Data Availability Statement: All relevant data are within the paper.

Funding: These authors have no support or funding to report.

Competing Interests: The authors have declared that no competing interests exist.
Galectin-3 (Gal-3) is an emerging biomarker in heart failure that is involved in fibrosis and inflammation. However, its potential value as a prognostic marker in atrial fibrillation (AF) is unknown. The aim of this study was to assess the impact of AF catheter ablation on Gal-3 and evaluate its prognostic impact for predicting rhythm outcome after catheter ablation.

\section{Methods}

Gal-3 was measured at baseline and after 6 months using specific ELISA. AF recurrences were defined as any atrial arrhythmia lasting longer than $30 \mathrm{sec}$ within 6 months after ablation.

\section{Results}

In $105 \mathrm{AF}$ patients ( $65 \%$ males, age $62 \pm 9$ years, $52 \%$ paroxysmal $\mathrm{AF}$ ) undergoing catheter ablation, Gal-3 was measured at baseline and after 6 months and compared with an AFfree control cohort ( $n=14,50 \%$ males, age $58 \pm 11$ years). Gal-3 was higher in AF patients compared with AF-free controls (7.8 \pm 2.9 vs. $5.8 \pm 1.8, \mathrm{ng} / \mathrm{mL}, \mathrm{p}=0.013)$. However, on multivariable analysis, $B M I(p=0.007)$ but not $A F(p=0.068)$ was associated with Gal-3. In the AF cohort, on univariable analysis higher Gal-3 levels were associated with female gender $(p=0.028)$, higher BMI $(p=0.005)$ and both CHADS2 $(p=0.008)$ and CHA2DS2-VASC

$(p=0.016)$ scores, however, on multivariable analysis only BMI remained significantly associated with baseline Gal-3 ( $p=0.016)$. Gal-3 was similar 6 months after AF catheter ablation and was not associated with sinus rhythm maintenance. 


\section{Conclusions}

Although galectin-3 levels are higher in AF patients, this is driven by cardiometabolic comorbidities and not heart rhythm. Gal-3 is not useful for predicting rhythm outcome of catheter ablation.

\section{Introduction}

Several blood biomarkers have been identified which are helpful in supporting atrial fibrillation (AF) diagnosis, prognosis and outcomes. Since catheter ablation has become the cornerstone of non-pharmacological AF treatment the interest for blood biomarkers reflecting and predicting outcomes after this treatment strategy has rapidly grown [1].

Natriuretic peptides have previously been established for a variety of cardiovascular diseases as important markers of increased mortality and morbidity, e.g. in acute coronary syndromes, stable coronary disease or congestive heart failure. Recently, Hijazi et al. [2] demonstrated that elevation of NT-proBNP was independently related to increased risks of stroke and mortality in AF patients. Furthermore, baseline NT-proBNP level correlated with AF burden in patients with lone AF and independently predicted AF recurrences after catheter ablation [3]. Some studies demonstrated reduction of transcardiac BNP levels after AF catheter ablation emphasizing the significance of the left atrium as initial origin for BNP production in AF [4].

Because of its involvement in cardiac fibrosis, inflammation and remodelling processes, galectin-3 (Gal-3) is one of the emerging biomarkers in cardiac diseases [5]. Together with NTpro-BNP, Gal-3 demonstrated better prognostic value for mortality in general population and in patients with heart failure [6]. AF is associated with structural remodeling based on proinflammatory and pro-fibrotic changes in atrial tissue, thus suggesting an association between Gal-3 and AF. A recently published analysis of more than 3,000 participants of the Framingham Offspring cohort found that higher circulating Gal-3 concentrations were associated with increased risk of developing $\mathrm{AF}$ [7].

However, there are no systematic analyses of this biomarker in $\mathrm{AF}$ available and a possible association with rhythm outcomes after catheter ablation has not been investigated. Consequently, this study, for the first time, (i) compared Gal-3 in AF patients with a AF-free cohort, (ii) assessed trans-cardiac and trans-pulmonary gradients, (iii) assessed the impact of AF catheter ablation, and (iv) evaluated its potential prognostic impact for predicting rhythm outcome.

\section{Methods}

\section{Study population}

We recruited 105 patients with symptomatic AF who underwent radiofrequency catheter ablation at Heart Center Leipzig, Germany and fourteen AF-free controls matched for age, gender and heart disease. The study was approved by the local ethics committee (Medical Faculty, Leipzig University) and patients provided written informed consent for participation. Paroxysmal and persistent AF was defined according to current guidelines [8]. Paroxysmal AF was defined as self-terminating within 7 days after onset documented by previous routine electrocardiograms (ECG) or Holter ECG. Persistent AF was defined as any AF episode either lasting longer than 7 days or requiring drug or direct current cardioversion for termination. AF-free controls without AF-related symptoms were recruited from the outpatient clinic and freedom from $\mathrm{AF}$ was verified by previous ECG reports. 
In all $\mathrm{AF}$ patients, transthoracic and transesophageal echocardiography was performed prior to ablation. All class I or III antiarrhythmic medications with the exception of amiodarone were discontinued at least 5 half-lives before the procedure. Estimated glomerular filtration rate (eGFR) was calculated by using the Cockroft-Gault equation: (140-age) x weight $(\mathrm{kg}) \mathrm{x}$ (0.85 if female) / $72 \mathrm{x}$ serum creatinine $(\mathrm{mg} / \mathrm{dl})$.

\section{Radiofrequency catheter ablation}

Patients presenting with $\mathrm{AF}$ at the beginning of the procedure were electrically cardioverted and ablation was performed during sinus rhythm (i.e. AF termination with ablation was not attempted). Pulmonary vein (PV) isolation was performed by sequential application of radiofrequency energy at the antrum of the pulmonary veins. End-point was isolation of the PV with proof of both exit and entrance block. After the isolation of the pulmonary veins, electoanatomical voltage maps of the LA body in sinus rhythm were created in each patient using ablation catheter as a roving catheter. Potentials with amplitudes over $0.5 \mathrm{mV}$ were defined as normal, and potentials under $0.2 \mathrm{mV}$ as low-voltage. According to the underlying substrate and induced left atrial macro reentry tachycardias (LAMRT) additional lines transecting the scar areas to connect with healthy tissue or anatomical obstacles were ablated.

After ablation, class I and III antiarrhythmic drugs were not reinitiated. Proton pump inhibitors were added for 4 weeks. According to the current guidelines [8], oral anticoagulation was prescribed for 3-6 months after catheter ablation and depending on risk stratification of stroke using the $\mathrm{CHA}_{2} \mathrm{DS}_{2}$-VASc score thereafter.

\section{Follow-up}

All patients were followed in the outpatient clinic for 6 months after catheter ablation. During this follow-up period, 7-days Holter ECG recordings were performed (immediately, 3 and 6 months after the ablation). Additional ECGs and Holter ECG recordings were obtained when patients' symptoms were suggestive of AF. Early AF recurrences (ERAF) were defined as any atrial arrhythmia lasting $>30$ seconds within first week after catheter ablation, while late AF recurrences (LRAF) were any episodes between 3 and 6 months after ablation.

\section{Blood samples}

Blood samples were obtained before and 6 months after catheter ablation from peripheral vein. In a sub-group of 10 patients, additional blood samples were taken from cardiac circulation (coronary sinus and left atrium) before ablation.

Platelet-poor plasma fractions were obtained by centrifugation at $20^{\circ} \mathrm{C}$ for $10 \mathrm{~min}$ at $3500 \times \mathrm{g}$, and plasma were stored at $-80^{\circ} \mathrm{C}$ for subsequent analysis. Galectin-3 plasma levels were quantified using commercially available specific enzyme-linked immunoabsorbent assays (ELISA) according to manufacturer protocol (Hölzel Galectin-3 (human) ELISA HZ-4858, Cologne, Germany). Intra-assay coefficient of variation for ELISA assay was $<5 \%$, and inter-assay variance was $<10 \%$. Results were compared with standard curves and the lower detection limit was $0.12 \mathrm{ng} / \mathrm{ml}$.

\section{Statistical analysis}

Data are presented as mean and standard deviation (SD) or median [interquartile range] for continuous variables and as proportions for categorical variables. Comparison of continuous variables was performed using the unpaired Student's t-test and of categorical variables using the Pearson chi-square test. To investigate the relationship between Gal-3 plasma levels and 
clinical variables, linear regression was performed with Gal-3 plasma concentration as dependent variable (univariable analysis, UV). Logistic regression analysis was used to identify factors associated with AF recurrences after catheter ablation. Multivariable analyses (MV), which included variables with a p-value $<0.1$ found on univariable analysis, were performed to identify independent associations between clinical variables and baseline Gal-3 levels as well as rhythm outcomes. Comparison of biomarker plasma levels in the cardiac and peripheral circulation was performed using the Student's paired t-test to identify a potential transcardiac gradient, i.e. comparison of left atrium (LA) and coronary sinus (CS) levels, and to identify a potential transpulmonary gradient, i.e. comparison of LA and peripheral vein $(\mathrm{peV})$ levels.

A p-value $<0.05$ was considered as statistically significant. Statistical analyses were performed with SPSS statistical software version 17.

\section{Results}

\section{Gal-3 plasma levels in AF}

Baseline characteristics of the study population are summarized in Table 1 . There were no significant differences between AF and AF-free patients. AF patients had significantly higher Gal-3 levels than AF-free controls (7.8 \pm 2.9 vs. $5.8 \pm 1.8, \mathrm{ng} / \mathrm{mL}, \mathrm{p}=0.013$; Fig 1). However, on multivariable analysis, $\mathrm{BMI}(\mathrm{Beta}=.250, \mathrm{p}=0.007)$ but not $\mathrm{AF}(\mathrm{Beta}=.166, \mathrm{p}=0.068)$ was associated with Gal-3 levels (Table 2).

In the AF cohort, on univariable analysis higher Gal-3 levels were associated with female gen$\operatorname{der}($ Beta $=.165, \mathrm{p}=0.028)$, higher BMI $($ Beta $=.203, \mathrm{p}=0.005)$ and both $\mathrm{CHADS}_{2}($ Beta $=.174$, $\mathrm{p}=0.008)$ and $\mathrm{CHA}_{2} \mathrm{DS}_{2}$-VASC (Beta $\left.=.185, \mathrm{p}=0.016\right)$ scores. However, on multivariable analysis only BMI remained significantly associated with baseline Gal-3 levels (Beta $=.235$, $\mathrm{p}=0.016$ ) while female gender and both risk scores showed a trend for association (Table 3 ).

\section{Gal-3 levels in cardiac and peripheral circulation}

In 10 patients the levels between Gal-3 plasma concentrations in the left atrium (LA), coronary sinus (CS) were comparable with Gal-3 levels in peripheral vein (peV). Therefore, no transcardiac and trans-pulmonary gradients could be found (Fig 2).

Table 1. Baseline characteristics of the study population.

\begin{tabular}{llll}
\hline & AF patients $(\mathbf{n}=\mathbf{1 0 5})$ & AF-free controls $(\mathbf{n}=\mathbf{1 4})$ & $\boldsymbol{P}$-value \\
\hline Age, years & $62 \pm 9$ & $58 \pm 11$ & 0.105 \\
Males $(\%)$ & 65 & 50 & 0.282 \\
Paroxysmal AF $(\%)$ & 51 & - & - \\
Lone AF $(\%)$ & 71 & - & - \\
AF history, months & $60(2-540)$ & - & - \\
CHADS $_{2}$-Score & $1(0-4)$ & - & - \\
CHA $_{2}$ DS $_{2}$-VASc-Score & $2(0-7)$ & - & - \\
BMI, kg/m & $29 \pm 5$ & $26 \pm 4$ & 0.074 \\
LAD, mm & $42 \pm 6$ & $39 \pm 7$ & 0.115 \\
LVEF $(\%)$ & $60 \pm 8$ & $59 \pm 11$ & 0.787 \\
eGFR, ml/min & $97 \pm 30$ & $100 \pm 31$ & 0.759 \\
\hline
\end{tabular}

Abbreviations: BMI-body mass index; LAD—left atrial diameter; LVEF—left ventricular ejection fraction; eGFR-estimated glomerular filtration rate

Data are presented as median (interquartile range) or mean value \pm SD.

doi:10.1371/journal.pone.0123574.t001 


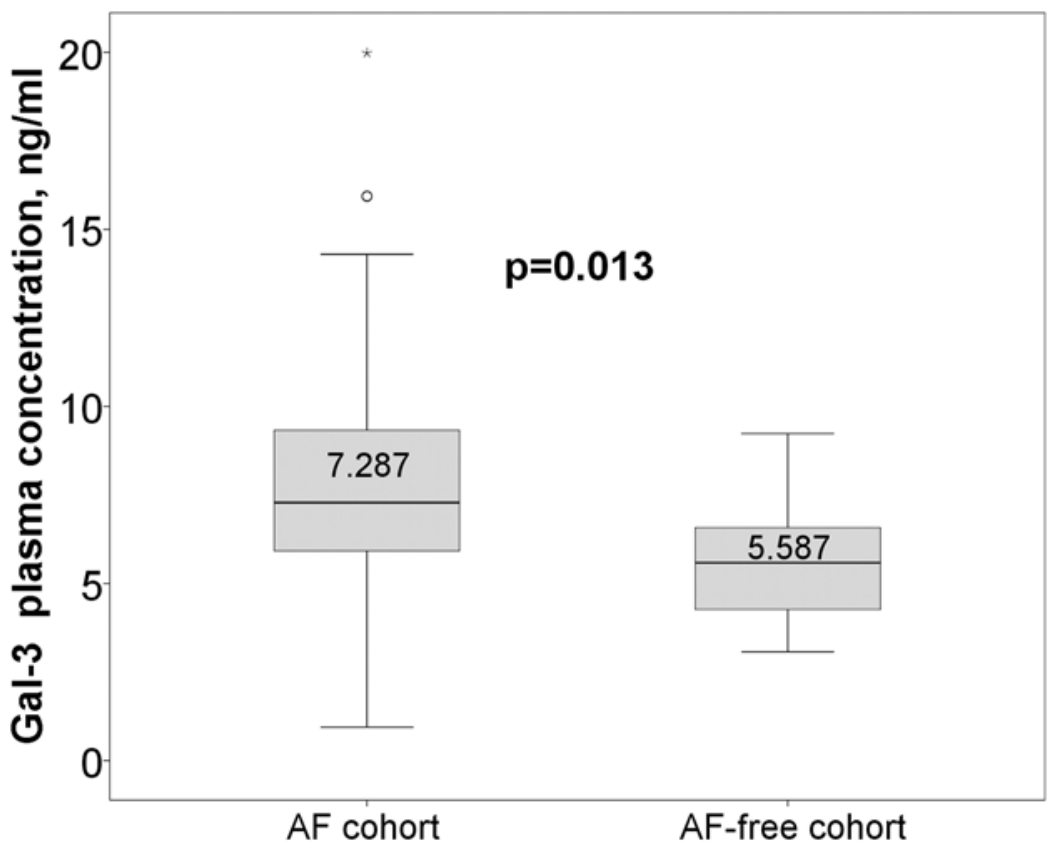

Fig 1. Gal-3 plasma levels in patients with AF and AF-free cohort.

doi:10.1371/journal.pone.0123574.g001

\section{Gal-3 as predictor for rhythm outcomes after catheter ablation}

Comparison of baseline characteristics and Gal-3 plasma levels in patients with and without AF recurrences is represented in Table 4. Patients with ERAF had more often persistent AF than patients without recurrence $(\mathrm{p}=0.04)$. There were no significant differences in clinical characteristics between patients with and without LRAF.

There were no significant differences in Gal-3 plasma levels in patients with and without ERAF ( $\mathrm{n}=0.382)$. Similar, Gal-3 levels at baseline and at 6 months were comparable in patients with and without LRAF (Fig 3). On multivariable analysis, only persistent AF (OR 2.84, 95\%CI $1.12-7.19, \mathrm{p}=0.003)$ and lone $\mathrm{AF}(\mathrm{OR} 0.29,95 \% \mathrm{CI} 0.11-0.80, \mathrm{p}=0.02)$ remained associated with AF recurrence.

Table 2. Clinical parameters associated with baseline Gal-3 plasma levels in study population.

\begin{tabular}{lclll}
\hline Variables & \multicolumn{2}{c}{ UV } & \multicolumn{2}{c}{ MV } \\
\cline { 2 - 3 } & Beta & P-value & Beta & P-value \\
\hline Age & .215 & 0.019 & .145 & 0.136 \\
Females & .180 & 0.051 & .120 & 0.212 \\
AF & .227 & 0.013 & .166 & 0.068 \\
BMI & .295 & 0.001 & .250 & 0.007 \\
Hypertension & .155 & 0.092 & .051 & 0.578 \\
LAD & .081 & 0.381 & & \\
LVEF & .025 & 0.785 & & \\
eGFR & -.097 & 0.303 & & \\
\hline
\end{tabular}

Abbreviations: as in Table 1; UV—univariable model, MV—multivariable model.

doi:10.1371/journal.pone.0123574.t002 
Table 3. Clinical parameters associated with baseline Gal-3 plasma levels in AF patients.

\begin{tabular}{|c|c|c|c|c|c|c|}
\hline \multirow[t]{2}{*}{ Variables } & \multicolumn{2}{|c|}{ UV } & \multirow{2}{*}{$\begin{array}{l}\text { MV* } \\
\text { Beta }\end{array}$} & \multirow{2}{*}{$\begin{array}{l}\text { Model } 1 \\
P \text {-value }\end{array}$} & \multirow{2}{*}{$\begin{array}{l}\text { MV } \\
\text { Beta }\end{array}$} & \multirow{2}{*}{$\begin{array}{l}\text { Model } 2 \\
P \text {-value }\end{array}$} \\
\hline & Beta & $P$-value & & & & \\
\hline Age & .167 & 0.088 & & & & \\
\hline Females & .215 & 0.028 & .165 & 0.081 & & \\
\hline Persistent AF & .076 & 0.438 & & & & \\
\hline BMI & .274 & 0.005 & .203 & 0.040 & .235 & 0.016 \\
\hline eGFR & -.080 & 0.422 & & & & \\
\hline LAD & .045 & 0.652 & & & & \\
\hline LVEF & .021 & 0.831 & & & & \\
\hline $\mathrm{CHADS}_{2}$ & .259 & 0.008 & .174 & 0.077 & & \\
\hline $\mathrm{CHA}_{2} \mathrm{DS}_{2}$-VASc & .234 & 0.016 & & & .185 & 0.055 \\
\hline
\end{tabular}

Abbreviations: as in Table 1 and 2.

*We performed multivariable analysis separately for $\mathrm{CHADS}_{2}$ and $\mathrm{CHA}_{2} \mathrm{DS}_{2}-\mathrm{VASc}$ score $\left(\mathrm{MV}\right.$ model 1 for the $\mathrm{CHADS}_{2}, \mathrm{MV}_{\text {model }} 2$ for the $\mathrm{CHA}_{2} \mathrm{DS}_{2}-$ VASc score).

doi:10.1371/journal.pone.0123574.t003

\section{Discussion}

\section{Main findings}

To the best of our knowledge, this study is the first to investigate the potential role of Gal-3 in $\mathrm{AF}$ and their response to catheter ablation. Several findings are of special importance: (i) Gal-3 levels are higher in AF patients than in AF-free controls, however, this seems to be mediated by higher BMI than by heart rhythm; (ii) there are no differences in Gal-3 trans-cardiac and trans-pulmonary levels; (iii) Gal-3 was not associated with early and late rhythm outcomes after AF catheter ablation.

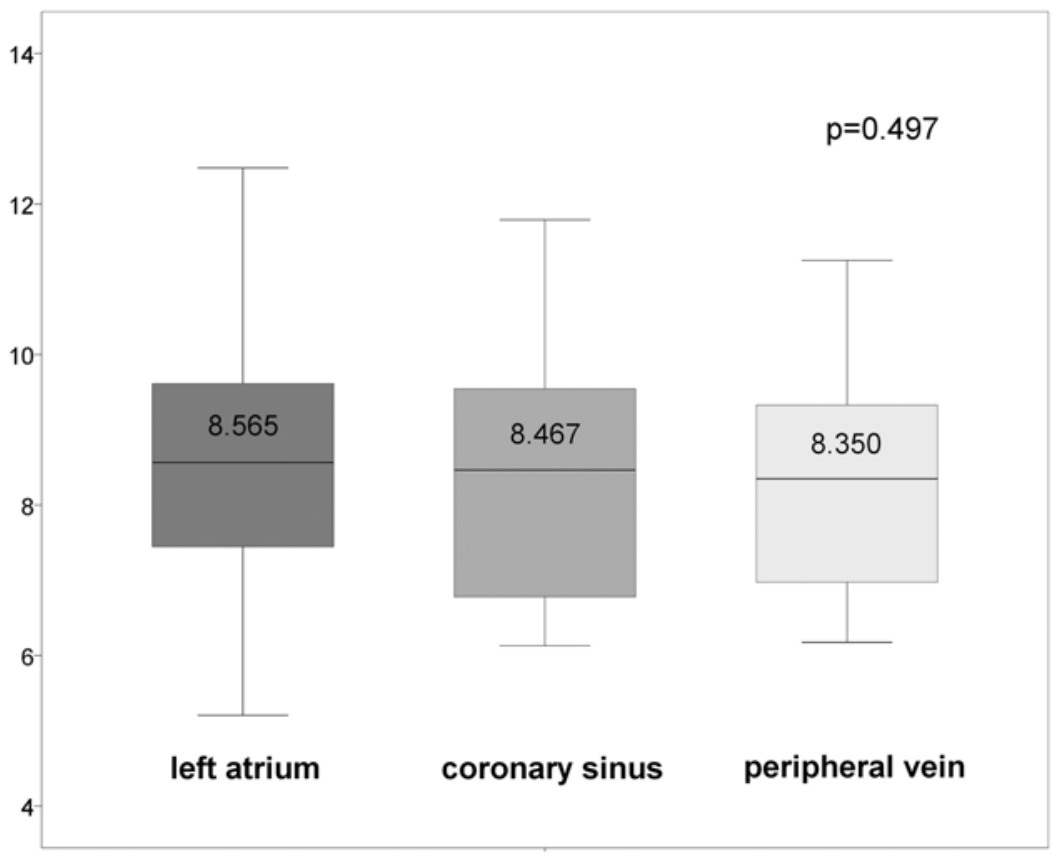

Fig 2. Gal-3 plasma levels in cardiac and peripheral circulation.

doi:10.1371/journal.pone.0123574.g002 
Table 4. Clinical characteristics and Gal-3 plasma levels at baseline and 6 months after catheter ablation in patients with and without AF recurrences.

\begin{tabular}{|c|c|c|c|c|c|c|}
\hline & \multicolumn{3}{|c|}{ ERAF* } & \multicolumn{3}{|c|}{ LRAF\# } \\
\hline & Yes $(n=40)$ & No $(n=55)$ & $P$-value & Yes $(n=36)$ & No $(n=56)$ & $P$-value \\
\hline Age, years & $61 \pm 9$ & $63 \pm 10$ & 0.382 & $63 \pm 9$ & $61 \pm 10$ & 0.429 \\
\hline Males (\%) & 68 & 66 & 0.835 & 69 & 64 & 0.609 \\
\hline Paroxysmal AF (\%) & 40 & 62 & 0.035 & 42 & 61 & 0.074 \\
\hline Lone AF (\%) & 68 & 76 & 0.339 & 61 & 80 & 0.043 \\
\hline AF history, months (median, IQR) & $60(2-480)$ & $60(3-540)$ & 0.888 & $36(2-216)$ & $60(3-540)$ & 0.181 \\
\hline $\mathrm{CHADS}_{2}$ (median, IQR) & $1(0-4)$ & $1(0-4)$ & 0.919 & $1(0-4)$ & $1(0-3)$ & 0.016 \\
\hline $\mathrm{CHA}_{2} \mathrm{DS}_{2}-\mathrm{VASc}$ (median, IQR) & $2(0-7)$ & $2(0-6)$ & 0.541 & $2(0-7)$ & $2(0-6)$ & 0.207 \\
\hline BMI & $29 \pm 5$ & $29 \pm 5$ & 0.687 & $30 \pm 6$ & $28 \pm 5$ & 0.156 \\
\hline $\mathrm{LAD}, \mathrm{mm}$ & $43 \pm 7$ & $42 \pm 6$ & 0.395 & $43 \pm 7$ & $41 \pm 6$ & 0.248 \\
\hline LVEF, \% & $60 \pm 10$ & $60 \pm 7$ & 0.708 & $60 \pm 11$ & $60 \pm 7$ & 0.901 \\
\hline eGFR, $\mathrm{ml} / \mathrm{min}$ & $100 \pm 37$ & $94 \pm 26$ & 0.343 & $95 \pm 36$ & $98 \pm 28$ & 0.671 \\
\hline Baseline Gal-3, ng/ml & $7.47 \pm 2.40$ & $8.01 \pm 3.37$ & 0.382 & $7.08 \pm 2.67$ & $8.09 \pm 3.15$ & 0.115 \\
\hline FU Gal-3, ng/ml & - & - & - & $7.09 \pm 2.75$ & $8.11 \pm 3.13$ & 0.111 \\
\hline
\end{tabular}

Abbreviations: as in the Table 1, ERAF-early AF recurrences, LRAF-late AF recurrences, FU—6 months follow-up.

*Data available in 95 patients

"Data available in 92 patients

doi:10.1371/journal.pone.0123574.t004

\section{Galectin-3 in AF}

Gal-3 had been shown as emerging biomarker for the prediction of mortality in patients with heart failure [6]. Because of inflammatory and pro-fibrotic processes leading to the structural remodeling in both diseases, heart failure is associated with $\mathrm{AF}$ and was even included into

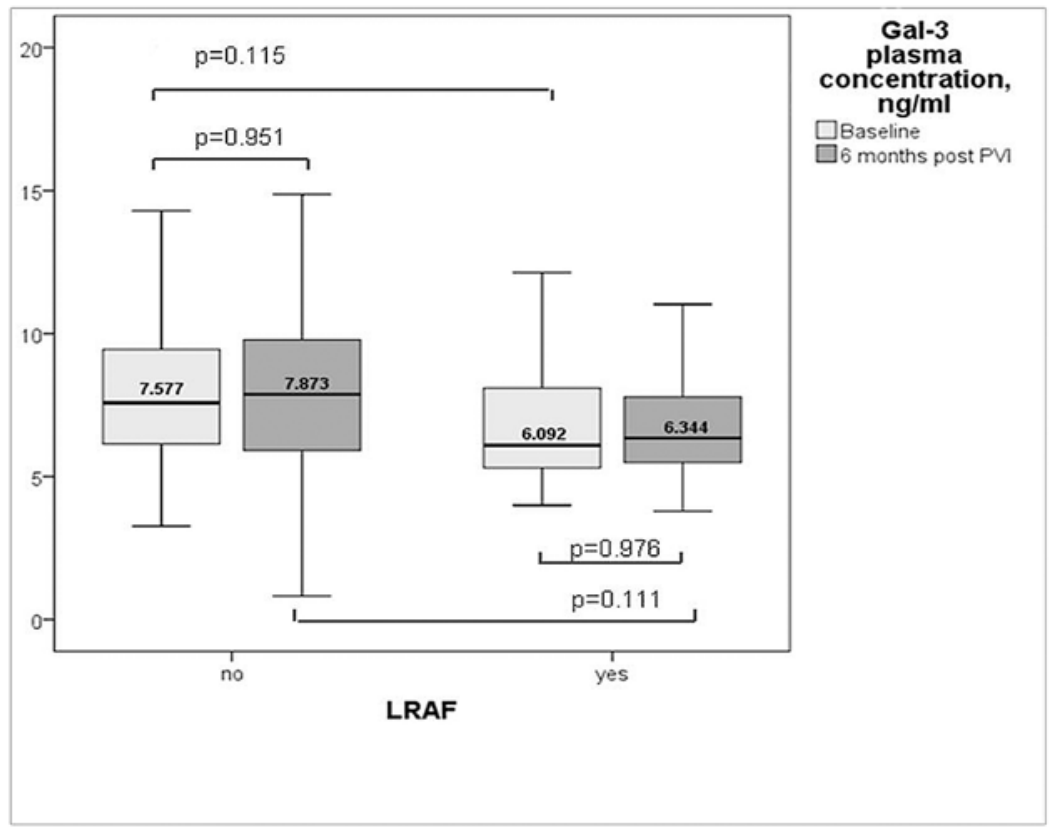

Fig 3. Gal-3 plasma levels in AF patients with and without AF recurrences.

doi:10.1371/journal.pone.0123574.g003 
thromboembolic risk stratification scores. However, the role of Gal-3 in AF is understudied. Recently, Ho et al [7] demonstrated an association between higher circulating Gal-3 concentrations and increased risk of developing AF, however, this study failed to demonstrate, that Gal-3 levels could be used to predict AF after adjustment for traditional clinical risk factors. De Boer et al [9] demonstrated that elderly population and women have higher Gal-3 levels that also was confirmed in our study. In contrast to recently published study by Gurses et al [10] we failed to demonstrate higher Gal-3 levels in patients with persistent AF. Although similarly to previous study we found higher Gal-3 levels in patients with AF than in controls, further analyses revealed the impact of underlying co-morbidity (reflected by the $\mathrm{CHADS}_{2}$ and $\mathrm{CHA}_{2} \mathrm{DS}_{2}$ VASc scores) than the rhythm per se. The gender related differences in Gal-3 levels might be explained by hormonal regulation while relation with other AF related co-morbidities (such as diabetes) has recently been investigated [11]. This study also demonstrated higher Gal-3 levels in obese patients that together with diabetes mellitus represent an important part of metabolic syndrome. We confirm these results by showing a significant association between higher BMI and baseline Gal-3 levels in both the entire and the AF cohort.

Several studies have compared biomarker levels in peripheral and cardiac circulation [4, 12]. For instance, inflammation is a known modulating factor in AF arrhythmogenesis and contributes to AF maintenance and perpetuation. Recently, Marcus et al demonstrated significantly lower levels of inflammatory markers in the coronary sinus than in the femoral vein in patients in atrial flutter, while patients in sinus rhythm showed no such difference. Thus, it had been suggested that atrial arrhythmias result in a collection of inflammatory cytokines in the heart, a process that could contribute to adverse remodeling [12].

Interestingly, in the non-failing heart, the source of BNP is believed to be in the atrium and BNP mRNA has been found in the left atrial wall [13]. Recently, it has been demonstrated that patients with paroxysmal AF have significantly higher BNP levels in cardiac circulation than AF-free controls [4]. Furthermore, ablation of the left atrium was associated with an immediate decrease of BNP levels, implicating this as the source. We also found higher Gal-3 levels in AF patients; however, differences in Gal-3 levels between the cardiac (coronary sinus and left atrium) and the peripheral circulation could not be found. A possible explanation could be the relation with systemic inflammation and/or collagen turnover rather than the cardiac source of these markers [14].

\section{Galectin-3 as predictor for the rhythm outcomes after catheter ablation}

Several studies were performed to identify clinical predictors for AF recurrences after catheter ablation. Among those persistent AF and enlarged left atrial diameter have reproducibly been shown to associate with AF recurrences [15]. Recently, in a large contemporary AF ablation cohort we found that all three stroke risk stratification scores (i.e. $\mathrm{CHADS}_{2}, \mathrm{CHA}_{2} \mathrm{DS}_{2}-\mathrm{VASc}$ and $\mathrm{R}_{2} \mathrm{CHADS}_{2}$ ) were significantly associated with rhythm outcomes within 12 months after AF catheter ablation [16].

Numerous studies evaluated the impact of inflammatory and pro-fibrotic markers in the prediction of rhythm outcomes after catheter ablation. Inflammatory markers, as a part of oxidative stress, can be related to both early and late AF recurrences after catheter ablation [17]. Recently, we indicated the possible role between inflammatory and auto-immune involvement and AF maintenance and progression, demonstrating an association between HSP70 and antiHSP70 antibodies with early and late recurrences after catheter ablation [18]. Of interest, circulating HSP70 may act as a pro-fibrotic regulator of the extracellular matrix proteins synthesis through changes in the cytokine TGF- $\beta 1$ production [19]. 
BNP (B-type natriuretic peptide) is a neurohormone secreted from the myocytes as response to increased wall tension by volume or pressure overload. It has been shown that baseline NT-proBNP levels are an independent predictor of AF recurrence after catheter ablation [3]. Interestingly, in patients with lone AF BNP levels correlated with AF burden and were also strongly associated with recurrent arrhythmia after ablation [20]. However, despite close relation of BNP and Gal-3 as markers for cardiovascular remodeling, we did not find the association between baseline Gal-3 levels and rhythm outcomes after catheter ablation. A possible explanation could be that both biomarkers might have different levels of sensitivity concerning cardiac rhythm. It has been already demonstrated that Gal-3 had a lower specificity and sensitivity to identify acute heart failure patients compared to NT-proBNP [21]. Furthermore, there are some differences in the pathophysiological mechanisms underlying the secretion of both biomarkers. Whereas BNP act as "loading biomarker", representing volume or pressure overload in the atria, Gal-3 is associated with turnover of the extracellular matrix and inflammation [22]. Therefore, Gal-3 plasma levels, measured in our study, are more likely reflecting a symbiosis of different cofounding variables (as age, gender, co-morbidities) as the cardiac rhythm per se also after catheter ablation.

\section{Limitations}

This study has several limitations. First, the study cohort included 105 consecutive Caucasian patients with different AF forms. Furthermore, our control group was relatively small and might be not sufficient to demonstrate significant differences in Gal-3 levels in patients with and without AF. Similar, the sub-group with different blood compartments was small and not significant or borderline results found in our population should be interpreted with caution but such sample sizes are usually used in first exploratory studies [23]. The Gal-3 levels found in our study were lower than in the study by Ho et al [7]. This might be partly explained by different assays and very high participant number. However, despite this inconsistence, another studies reported similar or even lower Gal-3 levels $[10,11]$.

Monitoring of AF recurrence was limited to serial 7-day Holter ECGs which is in line with current guidelines but this strategy may nevertheless have missed asymptomatic AF recurrences. Despite several definitions for early AF recurrences, in current study ERAF was considered as any atrial arrhythmia lasting longer than $30 \mathrm{sec}$ and occurring within first week after ablation. Although the rate of ERAF could be underestimated due to non-inclusion of AF recurrences within the whole 3 months blanking period, from a practical point this seems to be an ideal scenario since alterations in management can be provided quite early.

Finally, this analysis was restricted to the measurement of Gal-3 and did not include other biomarkers.

\section{Conclusions}

Although Gal-3 levels are higher in AF patients, this is driven cardiometabolic co-morbidities and not heart rhythm. Gal-3 is not useful for predicting rhythm outcome of catheter ablation. Further studies are needed to define a potential role of Gal-3 in the AF population.

\section{Author Contributions}

Conceived and designed the experiments: JK VA AB. Performed the experiments: JS LU. Analyzed the data: JK JS VA AB. Contributed reagents/materials/analysis tools: JK VA AB. Wrote the paper: JK JS AB. Data collection, patient recruitment: SJ SD GH DH BD. 


\section{References}

1. Kornej J, Husser D, Bollmann A, Lip GY. Rhythm outcomes after catheter ablation of atrial fibrillation. Clinical implication of biomarkers. Hamostaseologie 2014; 34:9-19. doi: 10.5482/HAMO-13-09-0051 PMID: 24166596

2. Hijazi Z, Oldgren J, Andersson U, Connolly SJ, Ezekowitz MD, Hohnloser SH, et al. Cardiac biomarkers are associated with an increased risk of stroke and death in patients with atrial fibrillation: a Randomized Evaluation of Long-term Anticoagulation Therapy (RE-LY) substudy. Circulation 2012; 125:1605-1616. doi: 10.1161/CIRCULATIONAHA.111.038729 PMID: 22374183

3. den Uijl DW, Delgado V, Tops LF, Ng AC, Boersma E, Trines SA, et al. Natriuretic peptide levels predict recurrence of atrial fibrillation after radiofrequency catheter ablation. Am Heart J 2011; 161:197-203. doi: 10.1016/j.ahj.2010.09.031 PMID: 21167354

4. Gould PA, Gula LJ, Bhayana V, Subbiah RN, Bentley C, Yee R, et al. Characterization of cardiac brain natriuretic peptide release in patients with paroxysmal atrial fibrillation undergoing left atrial ablation. Circ Arrhythm Electrophysiol 2010; 3:18-23. doi: 10.1161/CIRCEP.108.831586 PMID: 19966014

5. Yu L, Ruifrok WP, Meissner M, Bos EM, van Goor H, Sanjabi B, et al. Genetic and pharmacological inhibition of galectin-3 prevents cardiac remodeling by interfering with myocardial fibrogenesis. Circ Heart Fail 2013; 6:107-117. doi: 10.1161/CIRCHEARTFAILURE.112.971168 PMID: 23230309

6. van Kimmenade RR, Januzzi JL Jr., Ellinor PT, Sharma UC, Bakker JA, Low AF, et al. Utility of aminoterminal pro-brain natriuretic peptide, galectin-3, and apelin for the evaluation of patients with acute heart failure. J Am Coll Cardiol 2006; 48:1217-1224. PMID: 16979009

7. Ho JE, Yin X, Levy D, Vasan RS, Magnani JW, Ellinor PT, et al. Galectin 3 and incident atrial fibrillation in the community. Am Heart J 2014; 167:729-734 e721. doi: 10.1016/j.ahj.2014.02.009 PMID: 24766984

8. Camm AJ, Lip GY, De Caterina R, Savelieva I, Atar D, Hohnloser SH, et al. 2012 focused update of the ESC Guidelines for the management of atrial fibrillation: an update of the 2010 ESC Guidelines for the management of atrial fibrillation. Developed with the special contribution of the European Heart Rhythm Association. Eur Heart J 2012; 33:2719-2747. doi: 10.1093/eurheartj/ehs253 PMID: 22922413

9. de Boer RA, Edelmann F, Cohen-Solal A, Mamas MA, Maisel A, Pieske B. Galectin-3 in heart failure with preserved ejection fraction. Eur J Heart Fail 2013; 15:1095-1101. doi: 10.1093/eurjhf/hft077 PMID: 23650131

10. Gurses KM, Yalcin MU, Kocyigit D, Canpinar H, Evranos B, Yorgun H, et al. Effects of Persistent Atrial Fibrillation on Serum Galectin-3 Levels. Am J Cardiol 2014

11. Weigert J, Neumeier M, Wanninger J, Bauer S, Farkas S, Scherer MN, et al. Serum galectin-3 is elevated in obesity and negatively correlates with glycosylated hemoglobin in type 2 diabetes. J Clin Endocrinol Metab 2010; 95:1404-1411. doi: 10.1210/jc.2009-1619 PMID: 20080851

12. Marcus GM, Smith LM, Glidden DV, Wilson E, McCabe JM, Whiteman D, et al. Markers of inflammation before and after curative ablation of atrial flutter. Heart Rhythm 2008; 5:215-221. doi: 10.1016/j.hrthm. 2007.10.007 PMID: 18242542

13. Doyama K, Fukumoto M, Takemura G, Tanaka M, Oda T, Hasegawa K, et al. Expression and distribution of brain natriuretic peptide in human right atria. J Am Coll Cardiol 1998; 32:1832-1838. PMID: 9857859

14. Marcus GM, Smith LM, Ordovas K, Scheinman MM, Kim AM, Badhwar N, et al. Intracardiac and extracardiac markers of inflammation during atrial fibrillation. Heart Rhythm 2010; 7:149-154. doi: 10.1016/j. hrthm.2009.10.004 PMID: 20022819

15. D'Ascenzo F, Corleto A, Biondi-Zoccai G, Anselmino M, Ferraris F, di Biase L, et al. Which are the most reliable predictors of recurrence of atrial fibrillation after transcatheter ablation?: a meta-analysis. Int J Cardiol 2013; 167:1984-1989. doi: 10.1016/j.ijcard.2012.05.008 PMID: 22626840

16. Kornej J, Hindricks G, Kosiuk J, Arya A, Sommer P, Husser D, et al. Comparison of CHADS2, R2CHADS2 and CHA2DS2-VASc scores for prediction of rhythm outcomes after catheter ablation of atrial fibrillation: the Leipzig Heart Center AF Ablaiton Registry. Circ Arrhythm Electrophysiol 2014; 7:281-287. doi: 10.1161/CIRCEP.113.001182 PMID: 24610790

17. Kornej J, Reinhardt C, Kosiuk J, Arya A, Hindricks G, Adams V, et al. Response of high-sensitive C-reactive protein to catheter ablation of atrial fibrillation and its relation with rhythm outcome. PLoS One 2012; 7:e44165. doi: 10.1371/journal.pone.0044165 PMID: 22957001

18. Kornej J, Reinhardt C, Kosiuk J, Arya A, Hindricks G, Adams V, et al. Response of circulating heat shock protein 70 and anti-heat shock protein 70 antibodies to catheter ablation of atrial fibrillation. J TransI Med 2013; 11:49. doi: 10.1186/1479-5876-11-49 PMID: 23432758

19. Gonzalez-Ramos M, Calleros L, Lopez-Ongil S, Raoch V, Griera M, Rodriguez-Puyol M, et al. HSP70 increases extracellular matrix production by human vascular smooth muscle through TGF-beta1 upregulation. Int J Biochem Cell Biol 2013; 45:232-242. doi: 10.1016/j.biocel.2012.10.001 PMID: 23084979 
20. Hussein AA, Saliba WI, Martin DO, Shadman M, Kanj M, Bhargava M, et al. Plasma B-type natriuretic peptide levels and recurrent arrhythmia after successful ablation of lone atrial fibrillation. Circulation 2011; 123:2077-2082. doi: 10.1161/CIRCULATIONAHA.110.007252 PMID: 21536999

21. van Kimmenade R, van Dielen F, Bakker J, Nijhuis J, Crijns H, Buurman W, et al. Is brain natriuretic peptide production decreased in obese subjects? J Am Coll Cardiol 2006; 47:886-887. PMID: 16487862

22. de Boer RA, Yu L, van Veldhuisen DJ. Galectin-3 in cardiac remodeling and heart failure. Curr Heart Fail Rep 2010; 7:1-8. doi: 10.1007/s11897-010-0004-x PMID: 20425490

23. Okumura $Y$, Watanabe I, Nakai T, Ohkubo K, Kofune T, Kofune M, et al. Impact of biomarkers of inflammation and extracellular matrix turnover on the outcome of atrial fibrillation ablation: importance of matrix metalloproteinase-2 as a predictor of atrial fibrillation recurrence. J Cardiovasc Electrophysiol 2011; 22:987-993. doi: 10.1111/j.1540-8167.2011.02059.x PMID: 21489023 